Div. 562

NBSIR 80-2036R

\title{
Examination of Failed Six-Inch Diameter Cast-Iron Pipe Water Main, Philadelphia, Pennsylvania
}

T. Robert Shives

Fracture and Deformation Divisicn Center for Materials Science National Measurement Laboratory National Bureau of Standards U.S. Department of Commerce Washington, D.C. 20234

January 1980

Issued April 1980

Failure Analysis Report

\section{Prepared for}

National Transportation Safety Board Washington, D.C. 20594 



\section{EXAMINATION OF FAILED SIX-INCH \\ DIAMETER CAST-IRON PIPE WATER \\ MAIN, PHILADELPHIA, PENNSYLVANIA}

T. Robert Shives

Fracture and Deformation Division

Center for Materials Science

National Measurement Laboratory

National Bureau of Standards

U.S. Department of Commerce

Washington, D.C. 20234

January 1980

Issued April 1980

Failure Analysis Report

Prepared for

National Transportation Safety Board

Washington, D.C. 20594

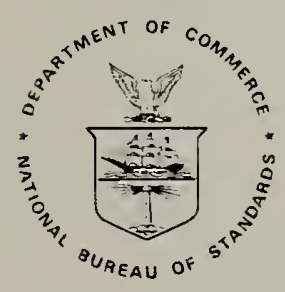

U.S. DEPARTMENT OF COMMERCE, Philip M. Klutznick, Secretary

Luther H. Hodges, Jr., Deputy Secretary

Jordan J. Baruch, Assistant Secretary for Productivity, Technology, and Innovation

NATIONAL BUREAU OF STANDARDS, Ernest Ambler, Director 



\section{SUMMARY}

1. INTRODUCTION
1.1 Reference
1.2 Background Information
1.3 Parts Submitted

2. PURPOSE

3. RESULTS OF EXAMINATIONS, TESTS, AND ANALYSES

3.1 Visual Examination of the Pipe Wall Surface

3.2 Fractographic Examination

3.3 Examination for Graphitization

3.4 Chemical Analysis

3.5 Hardness Measurements

3.6 Metallographic Examination

4. DISCUSSION

5. CONCLUSIONS

6. ACKNOWLEDGEMENT

REFERENCES

FIGURES

1. Piece of submitted pipe designated $W 7$ as received at NBS showing the fracture surface.

2. Piece of submitted pipe designated $W 2$ as received at NBS showing the fracture surface.

3. Pieces of submitted pipe designated $W 3$ as received at NBS showing the fracture surface.

4. Fracture surface W1 after cleaning. 
5. Fracture surface W2 after cleaning.

6. Fracture surface $W 3$ after cleaning.

7. SEM fractograph of W1.

8. SEM fractograph of $W 7$ at much higher magnification than figure 7 .

9. Transverse section through $W 7$ about $3 / 4$ inch from the fracture showing graphitization adjacent to the inside wall surface.

10. Transverse section through 22 about $3 / 4$ inch from the fracture showing graphitization adjacent to the inside wall surface.

11. Transverse section through 33 ranging from about $1 / 2$ to 2 inches from the fracture showing graphitization adjacent to both the inside and outside wall surfaces.

12. Etched longitudinal section through WI showing the fracture profile.

13. Unetched longitudinal section through Wi showing the fracture profile.

14. Etched longitudinal section showing the entire wall thickness of W3.

15. Etched longitudinal section through $W 7$ in a region which has not been graphitized.

16. Etched longitudinal section through $W 7$ in a region that has been essentially totally graphitized. 
SUMMARY

At the request of the National Transportation Safety Board, the National Bureau of Standards performed an examination of a fractured sixinch diameter cast-iron pipe water ma in from Tacony and Margaret Streets in Philadelphia, Pennsylvania. An explosion and fire had occurred at this location on May 13, 1979. At that time, both the six-inch diameter water main pipe and the eight-inch diameter cast-iron natural gas main pipe at the site of the explosion and fire were found to be completely fractured transversely in three places. Failure of the water pipe appeared to be due to an externally applied bending load.

The nonuniform wall thickness indicated that the pipe was pit cast and therefore rather old. Today, such pipe is normally centrifugally cast which results in a uniform wall thickness.

Each of the three fracture faces submitted for examination was covered with a rather tightly adhering layer of corrosion product. The corrosion product was not easily removed, and the corrosion process had obliterated some of the fracture features indicating that the fractures were not fresh. Cleavage was the predominant fracture mode. There was significant graphitization close to the fractures.

The hardness of the material was typical for gray cast iron. The chemical composition was typical for this material, but the phosphorus content was higher than desirable according to today's practice. The microstructure appeared to be satisfactory with the possible exception of more steadite than desirable according to today's practice. 

Examination of Failed Six-Inch Diameter Cast-Iron Pipe Water Main, Philadelphia, Pennsylvania

\section{INTRODUCTION}

\subsection{Reference}

National Transportation Safety Board, Washington, DC 20594. This investigation was conducted at the request of Mr. Jerry A. Houck, Metallurgist, National Transportation Safety Board. The requesting letter was dated June 25, 1979.

\subsection{Background Information}

The information in this section was furnished by the National Transportation Safety Board and by representatives of the Philadelphia Gas Works. On May 4, 1979, there was an explosion and fire at Tacony and Margaret Streets in Philadelphia, Pennsylvania. The explosion and fire were attributed to the ignition of an accumulation of natural gas at the site.

After the explosion and fire, both the eight-inch diameter cast-iron pipe natural gas main and the six-inch diameter cast-iron pipe water main at the site were found to be broken. The two pipelines were in close proximity and both lines had fractured completely in a circumferential manner in three places. (The results of an NBS examination of the failed gas pipe can be found in NBS Report NBSIR 80-1985, issued February, 1980).

When the pipelines in the region of the fractures were uncovered, the soil beneath the gas main was missing to a depth ranging from 3 inches to about 3 feet, 9 inches below the level where the pipeline had originally been installed. This missing soil created a cavity and left the gas pipeline unsupported. When the gas line fractured, it fell into this cavity.

\subsection{Parts Submitted}

Pieces of the water main pipe containing one fracture face from each of the three fractures were submitted to NBS for examination. The parts, designated $W 1$, W2, and $W 3$, are shown as received at NBS in figures 1,2 , and 3 , respectively. The pieces shown in figures 1 and 2 were complete, intact transverse sections. The pieces shown in figure 3 (W3) were from a transverse section that included the end of the pipe length. It was reported that this section was fractured longitudinally into the three pieces shown while being removed from the adjoining pipe length. The fracture in $W 7$ passed through the approximate center of a service tap hole in the pipe. 


\section{PURPOSE}

The National Transportation Safety Board requested that NBS determine the mode of failure for the broken water pipe.

\section{RESULTS OF EXAMINATIONS, TESTS, AND ANALYSES}

\subsection{Visual Examination of the Pipe Wall Surface}

Each piece of the submitted pipe was covered with a rather adherent layer of soil and some corrosion product. The pieces were cleaned ultrasonically, first with a commercial detergent, and then with buffered hydrochloric acid. Essentially all of the soil was removed from the outside of the pipe by the cleaning procedures. Some corrosion of both the inside and outside walls of the pipe was evident.

There was a lack of uniformity in the pipe wall thickness indicating that the pipe was pit cast, and therefore rather old. As an example of the nonuniform thickness, the wall thickness at the fracture in W1 ranged from about 0.43 inch minimum to about 0.53 inch maximum.

\subsection{Fractographic Examination}

Al1 three fracture surfaces were covered with corrosion product. The fracture surfaces were cleaned ultrasonically at the same time the pipe walls were cleaned. Some of the corrosion product adhered tightly to the fracture surfaces, and much of it remained on the surfaces after cleaning. The fact that the corrosion product was difficult to remove indicates that the pipe had probably been broken for some time. The cleaned surfaces of fractures $W 1$, W2, and W3 are shown respectively in figures 4,5 , and 6 .

Visually and macroscopically, all three fractures appeared similar. Therefore, a detailed fractographic examination was performed on only one of the fracture surfaces. After cleaning, part of the fracture surface from the piece of pipe designated W7 was examined with the scanning electron microscope (SEM). Many of the fracture features had been obiliterated by the corrosion process, but the fracture mode appeared to be predominantly cleavage. Cleavage indicates a very low ductility or brittle fracture, which would be expected for an overload fracture in gray cast iron.

A low magnification SEM fractograph is shown in figure 7 . Although some of the detail has been lost due to the corrosion process, and some is masked by the corrosion product that remained on the fracture surface, evidence of cleavage can still be seen. A much higher magnification SEM fractograph is shown in figure 8 . Corrosion product remaining on the fracture surface is quite evident. 
Transverse sections were taken close to each of the three fractures. These sections were polished metallographically to determine the extent of graphitization. The sections from W1, W2, and W3 are shown in figures 9, 10, and 11, respectively. Some graphitization adjacent to the inside wall surface is evident in each section, and in some regions, the depth of the graphitization is significant. In one region in the section from W1 (figure 9), graphitization starting at the inside had penetrated about $1 / 3$ of the pipe wall thickness.

The transverse sections from $W 1$ and W2 exhibited essentially no evidence of graphitization adjacent to the outside wall surface, but there was significant graphitization adjacent to the outside wall surface of W3 over most of the circumference of the section. This section is very close to the end of the pipe length.

\subsection{Chemical Analysis}

A sample from piece W2 was submitted to a commercial laboratory for chemical analysis. The results of that analysis are as follows:

Percent (by weight)

Element
Total carbon
Combined carbon
Manganese
Phosphorus
Sulfur
Sil icon
Nickel
Chromium
Molybdenum
Copper

Total carbon

Combined carbon

Manganese

Phosphorus

Sulfur

Silicon

Nickel

Chromium

Copper
3.21

1.01

0.42

1.97

0.084

1.86

0.04

0.01

$<0.01$

0.14

The carbon equivalent ${ }^{\top}$ ( $\%$ total carbon $+1 / 3$ ( $\%$ silicon $+\%$ phosphorus)) for the material is greater than $4.3 \%$, indicating that the material is hypereutectic and would therefore be expected to be relatively low in strength. Current ANSI Standard A21.6-1975 (American Water Works Association Standard (106-75) for cast iron pipe centrifugally cast in metal molds, for water or other 1iquids, and ANSI Standard 21.8-1975 (AWWA C108-75) for cast iron pipe centrifugally cast in sand-1ined molds, for water or other liquids, spec ify a maximum phosphorus content of $0.50 \%$ and a maximum sulfur content of $0.12 \%$. Since this pipe was pit cast, and therefore probably rather old, the standards quoted would not apply. They are cited for comparison only. Compared to today's practice, however, the phosphorus content is quite high. Excessive phosphorus leads to the formation of steadite (iron-iron phosphide eutectic). Steadite is hard and brittle, and if present in sufficient quantities, it may have adverse effects on the mechanical properties of the cast iron. 


\subsection{Hardness Measurements}

Rockwell K (HRK) hardness measurements were taken on the transverse section from $W 2$ that is shown in figure 10 . Ten measurements were made around the circumference. The range in hardness was HRK $851 / 2$ to 97 and the average value was 92.8. The approximate equivalent Brinell (HB) hardness values range from 136 to 176 and the approximate equivalent $H B$ average is 159. The variation in hardness is rather large, but the average hardness is typical for ordinary gray cast iron?.

\subsection{Metallographic Examination}

An etched longitudinal section through W1 showing the entire pipe wall thickness with the fracture profile horizontal at the top appears in figure 12. This section passes through a region of graphitization which can be seen adjacent to the inside wall surface at the left. An aspolished longitudinal section through wl showing part of the fracture profile at higher magnification appears in figure 13. The thin, dark particles are graphite flakes with an essentially type A distribution? A longitudinal section showing the entire wall thickness of W3 slightly removed from the fracture appears in figure 14 . This figure is at a lower magnification than figure 12. It should be noted that the region shown is very near the end of the pipe length where it connected to another pipe length while in service. There is significant graphitization adjacent to both the inside and outside wall surfaces.

An etched longitudinal section through $W 1$ in a region which has not been subject to graphitization is shown in figure 15 at much higher magnification than in figure 13. The microstructure consists of graphite flakes (long, thin, dark particles), pearlite (fingerprint pattern), ferrite (light gray patches), and probably steadite (small, rounded particles within the ferrite patches). A region in a longitudinal section through W1 exhibiting almost total graphitization is shown in figure 16 .

\section{DISCUSSION}

This six-inch diameter cast-iron pipe water main fractured completely in a circumferential manner in three places. There was corrosion product on the fracture surfaces. This corrosion product adhered rather well to the fractures, and some of the fracture features had been obliterated by corrosive attack. These two factors suggest that the pipeline had been fractured for some time. In contrast, the fracture examined from the failed cast-iron pipe natural gas main from the same location appeared to be relatively fresh (NBS report NBSIR 80-1985). The pipe failed in a brittle manner as expected for an overload fracture. Cleavage was the predominant fracture mode. The locations of the fracture origins were not established.

One of the fractures passed through the approximate center of a service tap hole in the pipe. This tap hole would have reduced the cross section of the pipe in the plane of the fracture which, in turn, would reduce the load carrying capacity of the pipe somewhat at this location. A significant amount of graphitization was found adjacent to the fractures. 
In a region near the end of the pipe length, there was graphitization adjacent to both the inside and outside wall surfaces of the pipe. Away from the end of the pipe, graphitization was found oniy adjacent to the inside wall surface. The graphitization would have also reduced the effective cross section of the pipe somewhat.

It appears that the pipe failed due to the application of an external bending load.

The chemical composition of the pipe material appeared to be satisfactory with the possible exception of phosphorus. The phosphorus content was higher than desirable according to today's practice. A high phosphorus content may lead to the formation of excessive steadite, a hard, brittle constituent. It is unclear whether the high phosphorus content was a contributing factor to the failure.

The hardness of the material varied considerably, but the average hardness was acceptable for gray cast-iron. The microstructure of the pipe material appeared to be satisfactory although some steadite was present.

\section{CONCLUSIONS}

1. This failed gray cast iron pipe water main had fractured completely

in three places in a transverse manner.

2. Failure appeared to be due to an external bending load applied to the pipe.

3. Much of the corrosion product adhered rather tightiy to the fracture surfaces and the corrosion process had obliterated some of the fracture features. These two factors suggest that the fractures had occurred some time ago.

4. The fracture mode was predominantly cleavage indicating that the fractures were brittle, which is normal for gray cast-iron.

5. There was significant graphitization in localized regions near the fractures.

6. The fracture origins were not established.

7. The chemical composition of the pipe material appeared to be typical for gray cast-iron with the possible exception of a higher phosphorus content than is usuat in today's grades of cast-iron.

8. The hardness of the material, although exhibiting considerable variation, was within the range typical for gray cast-iron.

9. The microstructure of the material appeared to be satisfactory, with the possible exception of more steadite than desirable. 


\section{ACKNOWLEDGEMENT}

The metallography and hardness measurements were performed by Leonard C. Smith of the NBS Fracture and Deformation Division. The photographic work was performed by Leonard Smith and Joel C. Sauter, also of the NBS Fracture and Deformation Division.

\section{REFERENCES}

1. Gray and Ductile Iron Castings Handbook, Gray and Ductile Iron Founder's Society, Inc., 1971.

2. Metals Handbook, 8th Edition, Volume 7, American Society for Metals, 1972. 


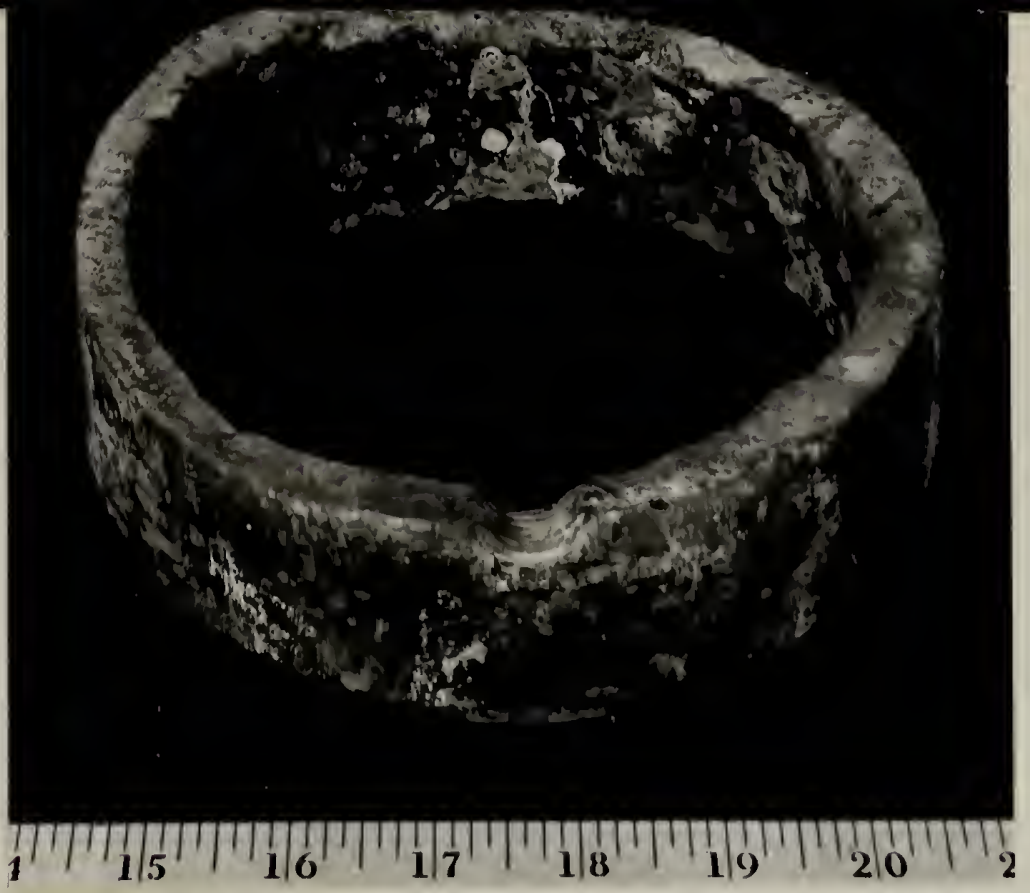

Figure 1. Piece of submitted pipe designated $W 1$ as received at NBS showing the fracture surface. The fracture passed through a service tap hóle.

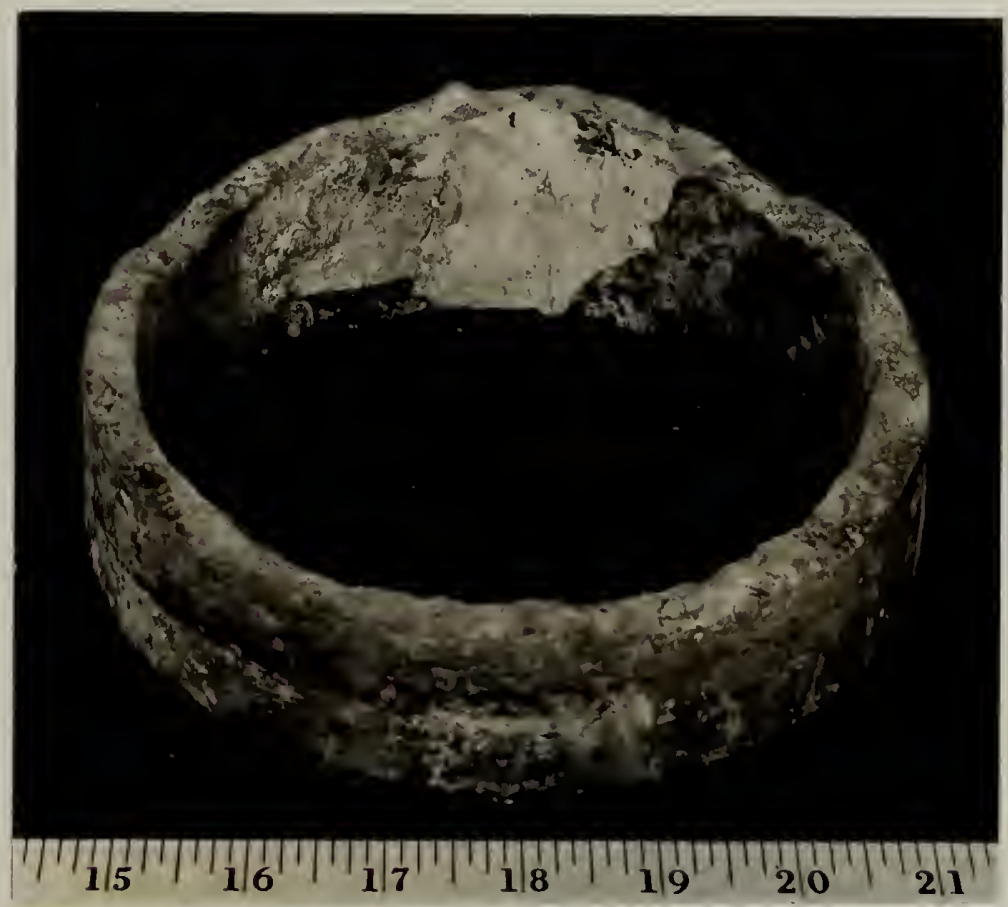

Figure 2. Piece of submitted pipe designated $W 2$ as received at NBS showing the fracture surface. 




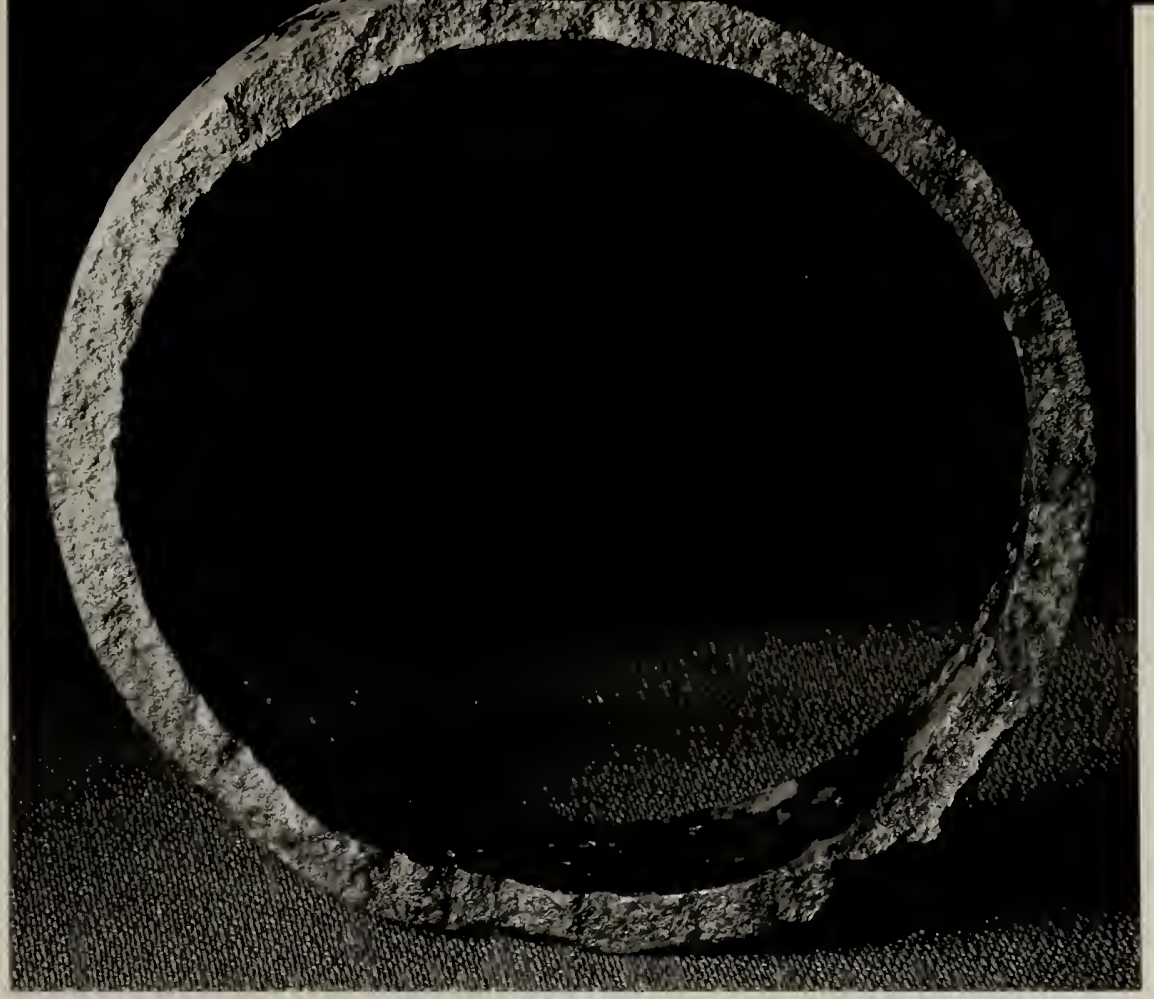

Figure 5. Fracture surface W2 after cleaning. $\quad \times 2 / 3$

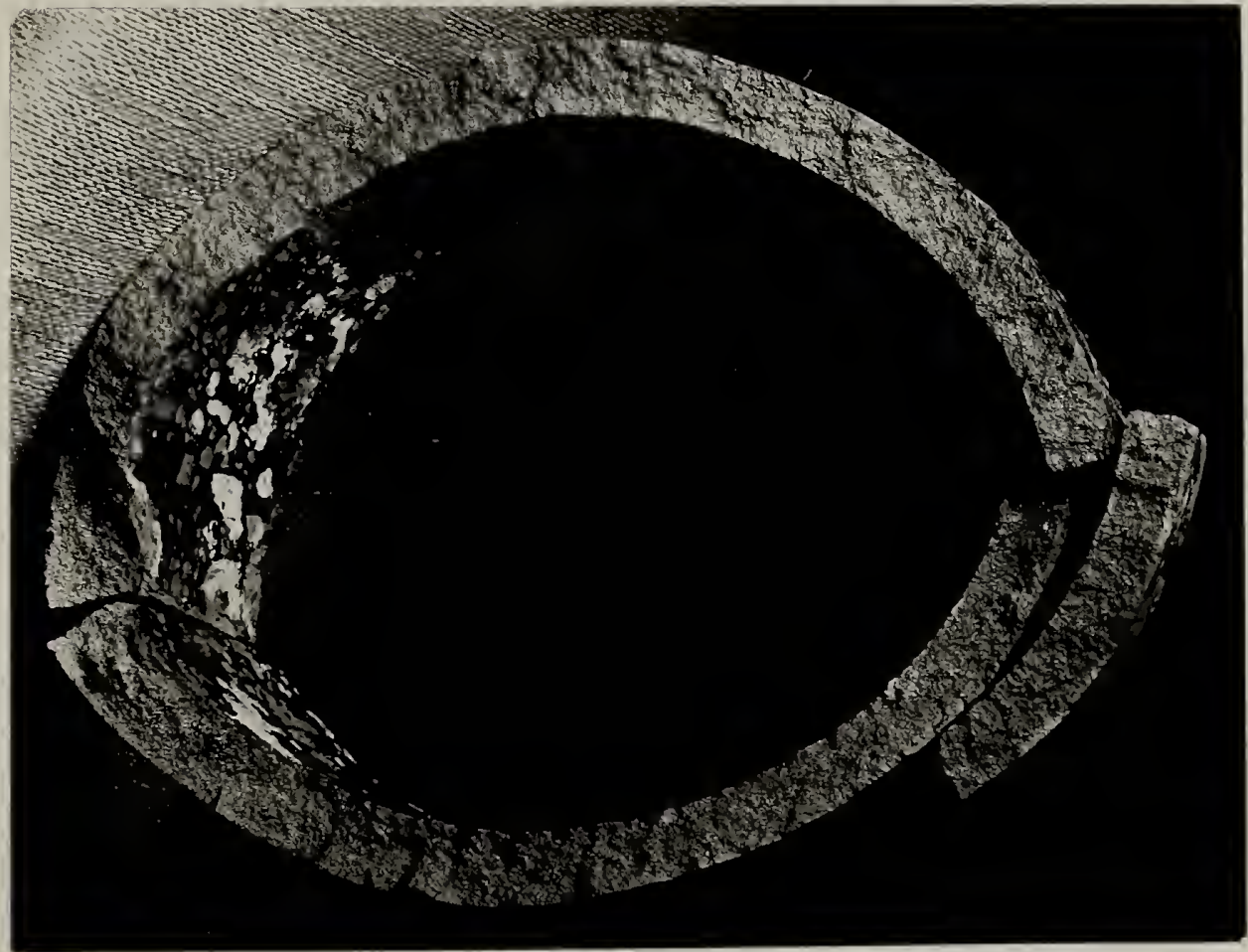

Figure 6. Fracture surface W3 after cleaning. $\quad \times 2 / 3$ 


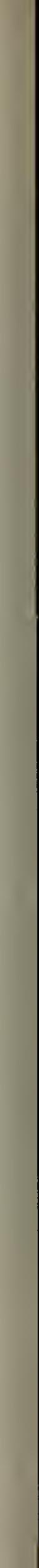




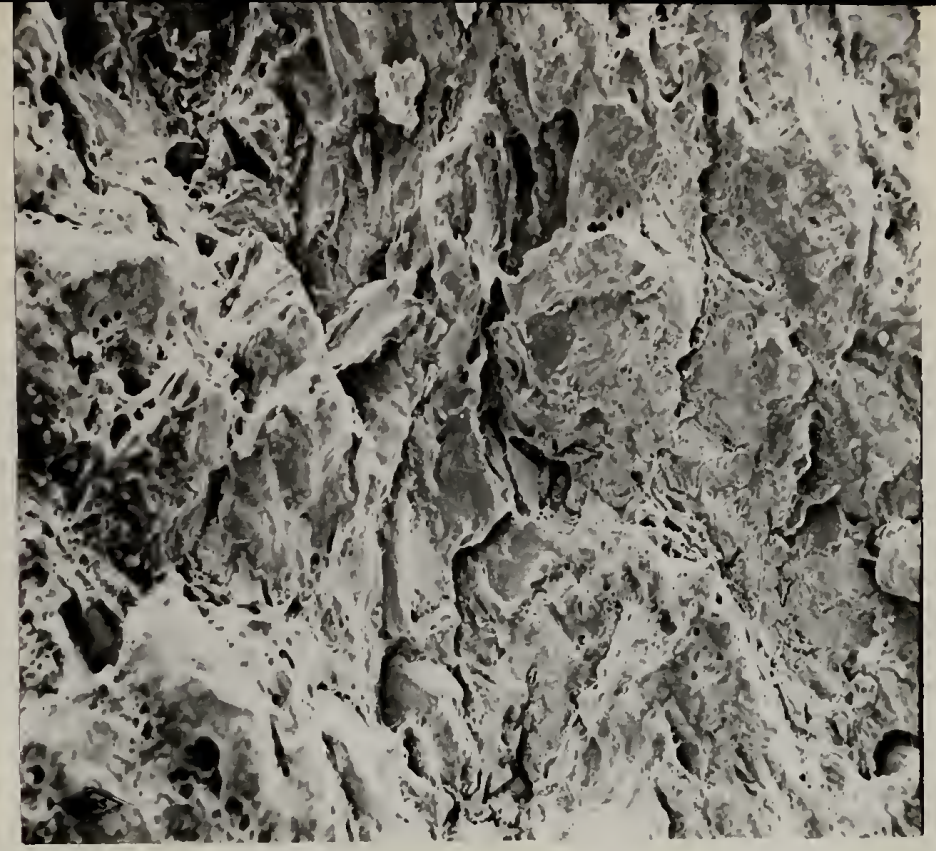

Figure 7. SEM fractograph of W1. The fracture mode is predominantly cleavage.

$\times 185$

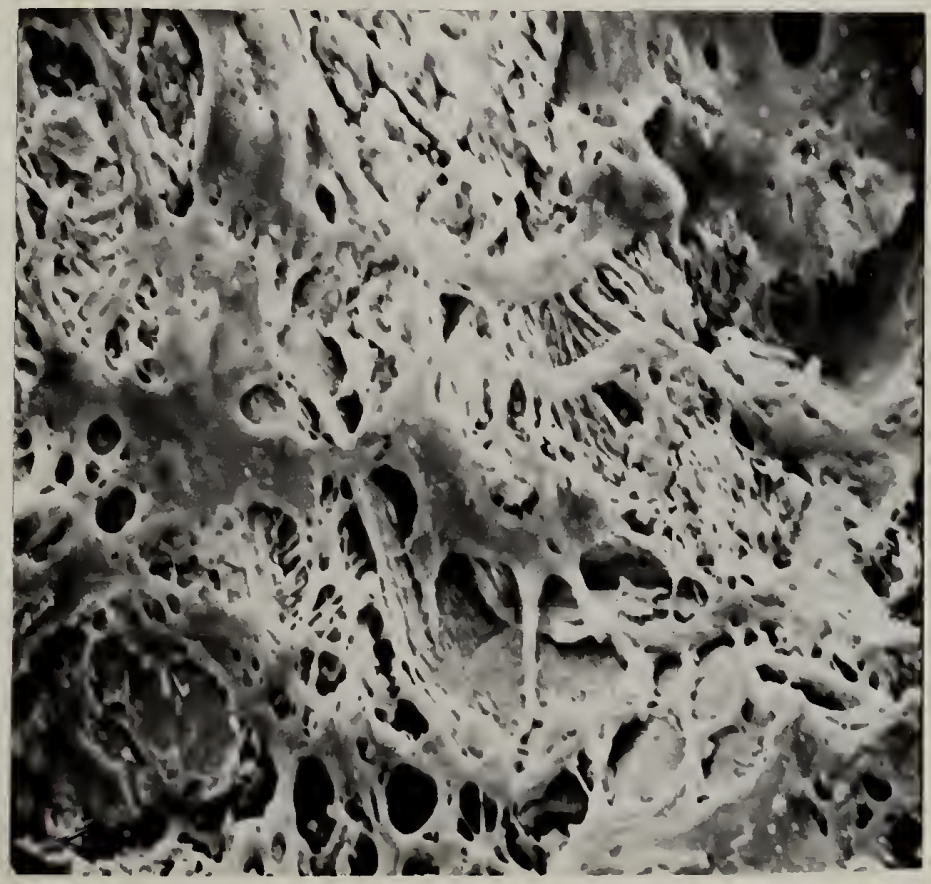

Figure 8. SEM fractograph of W1 at much higher magnification than figure 7 . Corrosion product is evident. $\times 1000$ 



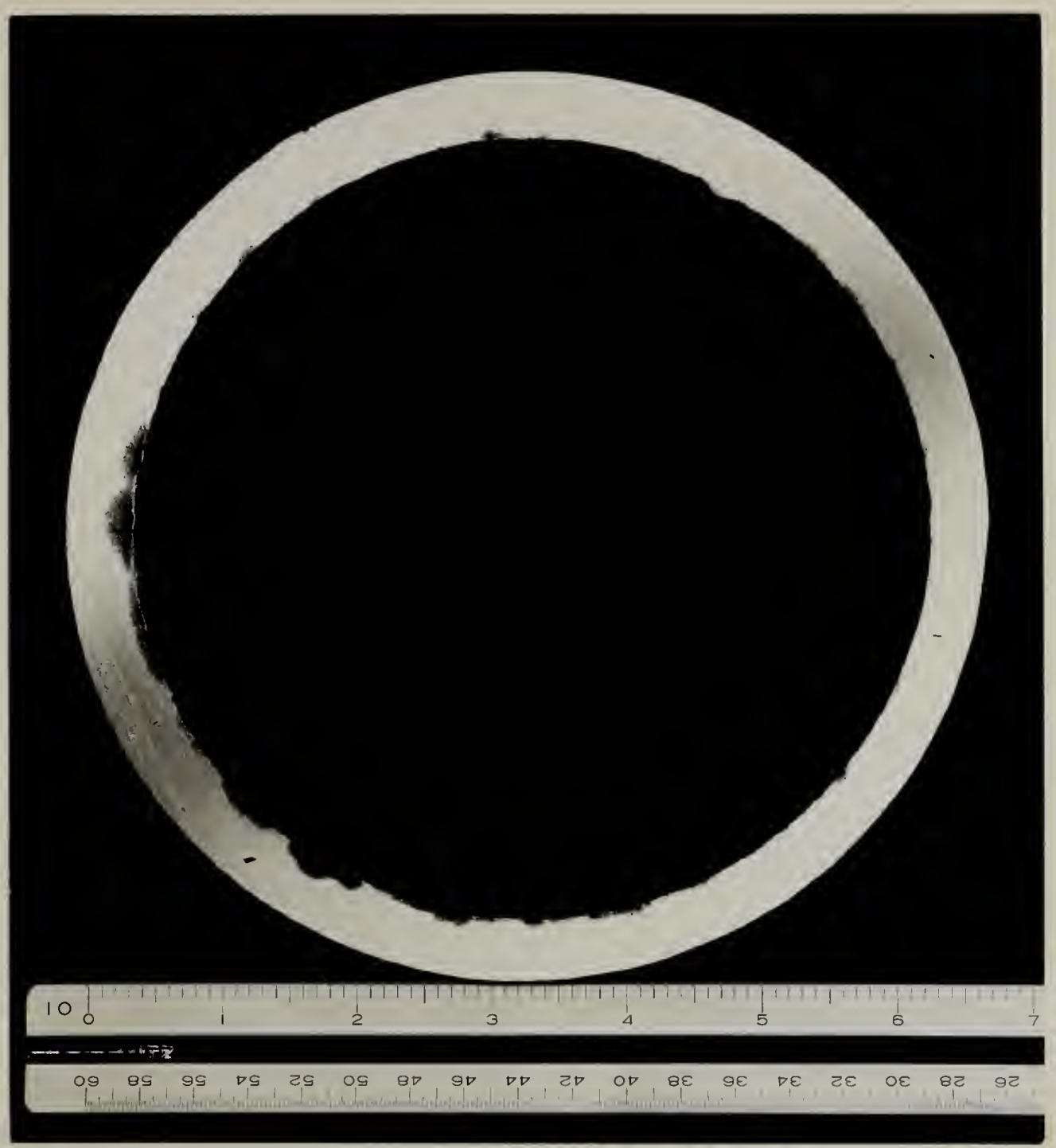

Figure 9. Transverse section through $W 7$ about $3 / 4$ inch from the fracture showing graphitization adjacent to the inside wall surface. $X 2 / 3$ 



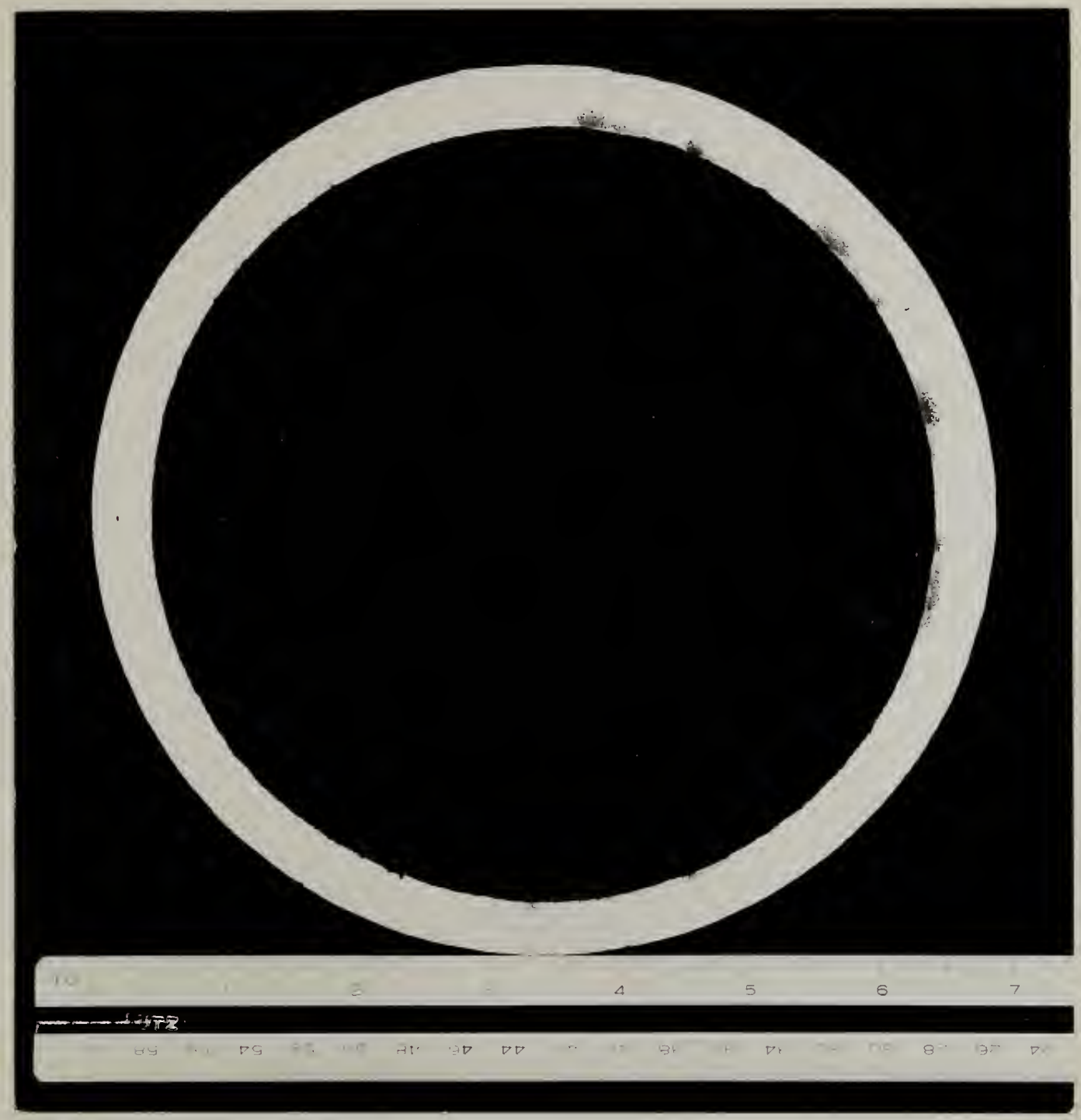

Figure 10. Transverse section through $W 2$ about $3 / 4$ inch from the fracture showing some graphitization adjacent to the inside wall surface. 



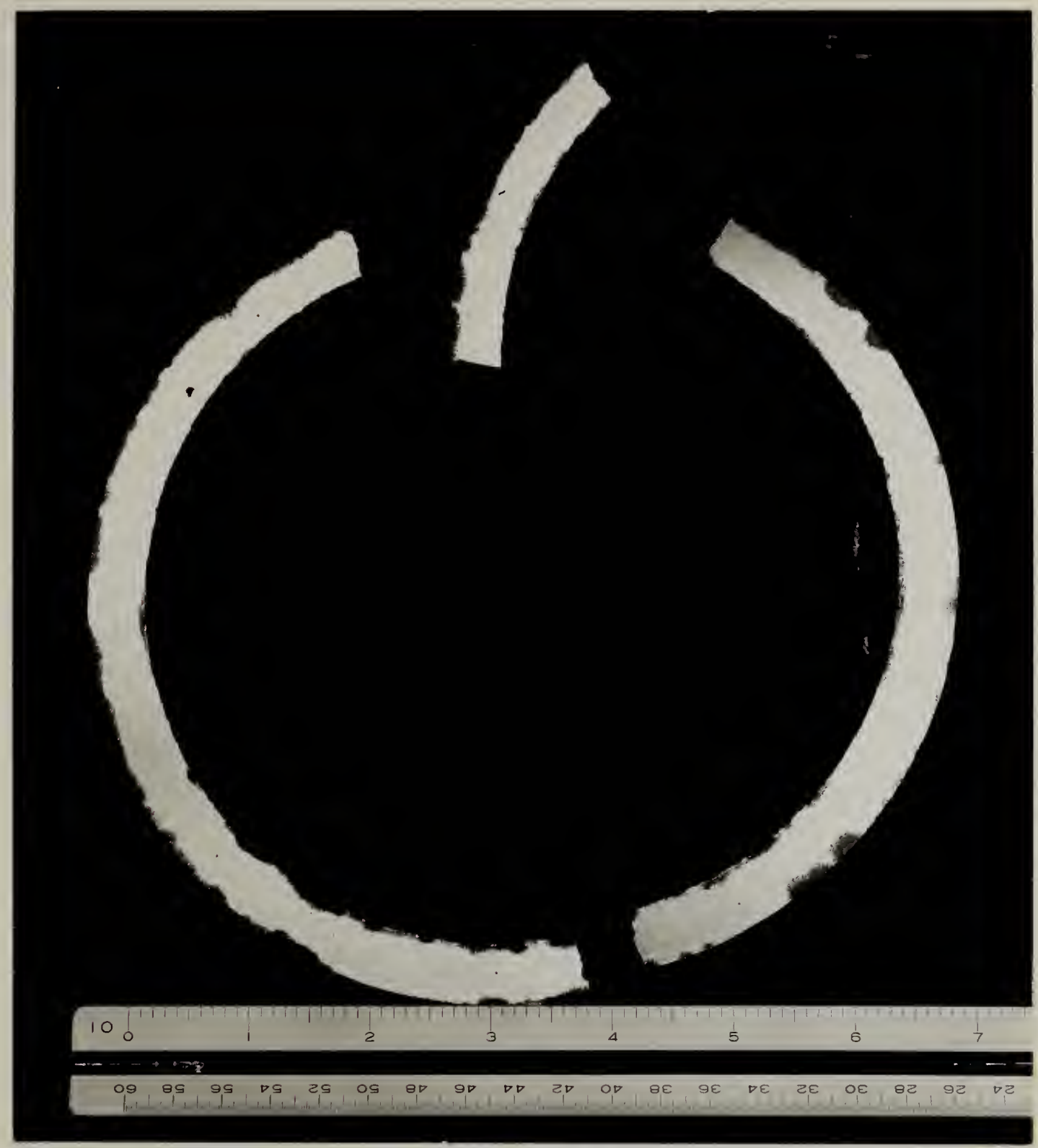

Figure 11. Transverse section through W3 ranging from about $1 / 2$ to 2 inches from the fracture showing graphitization adjacent to both the inside and outside wall surfaces. 



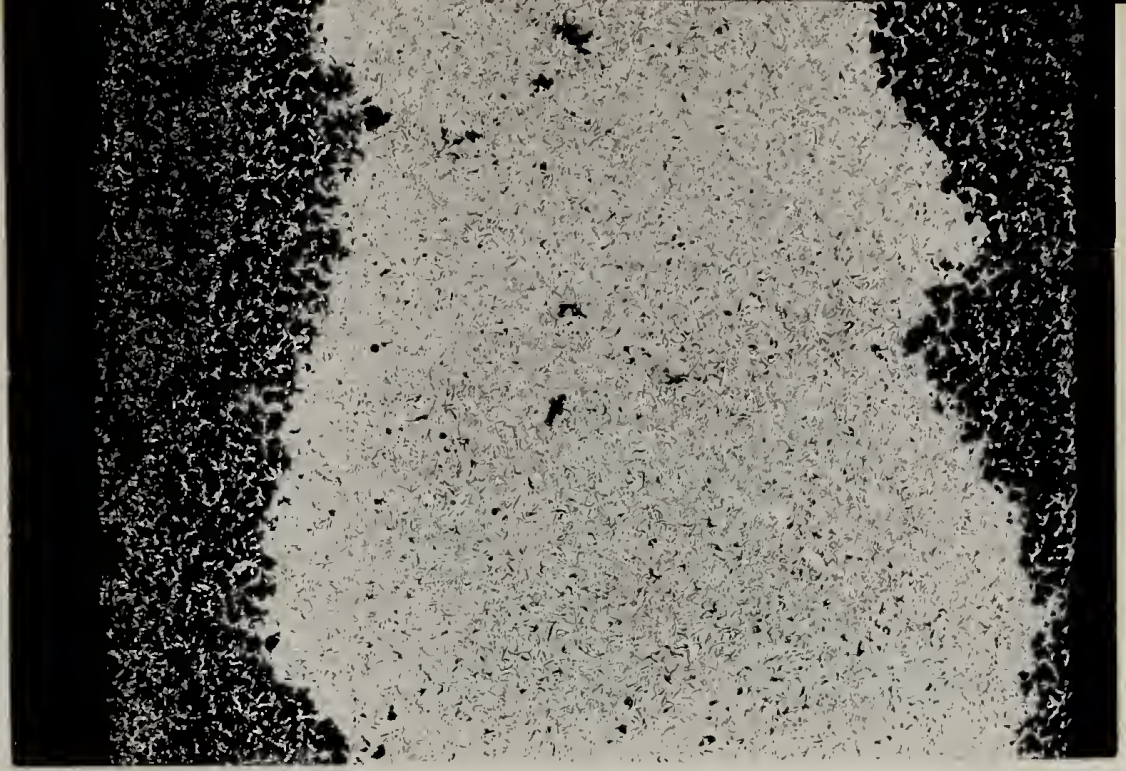

Figure 14. Etched longitudinal section showing the entire wall thickness of W3. Graphitization is evident both adjacent to the inside wall surface (left) and adjacent to the outside wall surface (right). Etchant: $1 \%$ nital $\times 8$

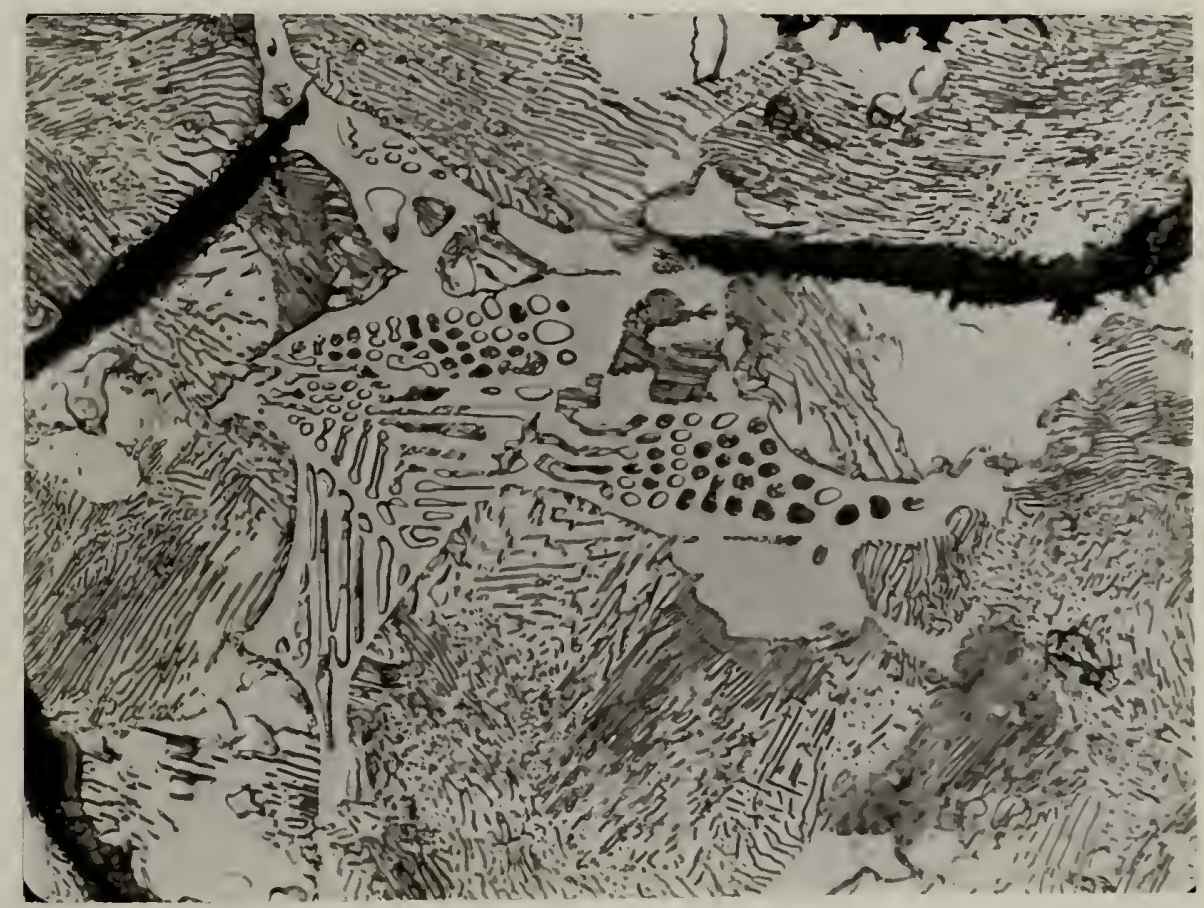

Figure 15. Etched longitudinal section through $W 1$ in a region which has not been graphitized. The microstructure consists of graphite flakes (long, thin dark particles), pearlite (fingerprint pattern), ferrite (1 ight gray patches), and probably steadite (small rounded particles within the ferrite patches). Etchant: $1 \%$ nital 



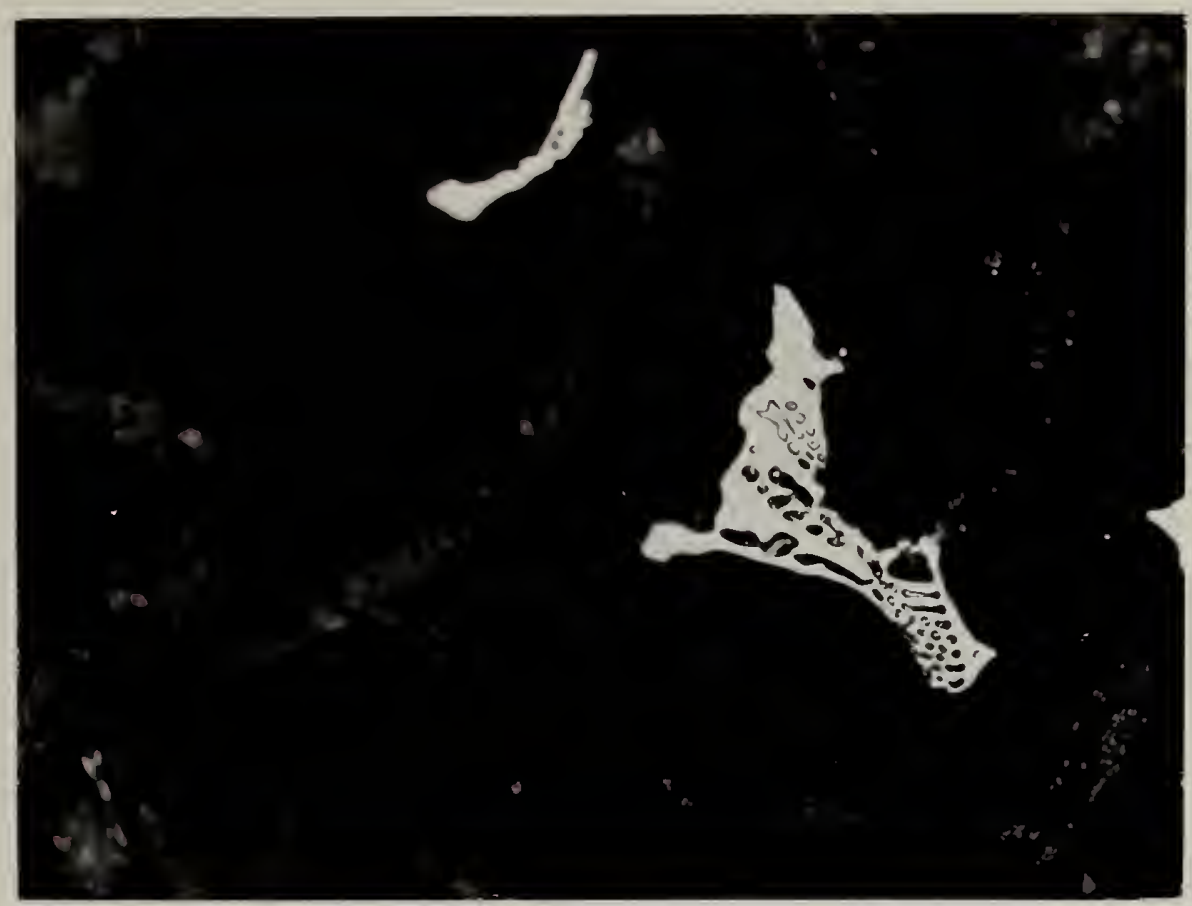

Figure 16. Etched longitudinal section through WT in a region that has been essentially totally graphitized.

Etchant: $1 \%$ nital $\quad \times 1000$ 



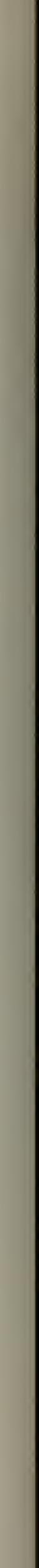

